\title{
High-energy neutrino signatures of dark matter
}

\author{
Matthew R. Buckley, ${ }^{1}$ Douglas Spolyar, ${ }^{2,3}$ Katherine Freese,${ }^{4}$ Dan Hooper, ${ }^{2,5}$ and Hitoshi Murayama ${ }^{6,7,8}$ \\ ${ }^{1}$ Department of Physics, California Institute of Technology, Pasadena, California 91125, USA \\ ${ }^{2}$ Center for Particle Astrophysics, Fermi National Accelerator Laboratory, Batavia, Illinois 60510 USA \\ ${ }^{3}$ University of California, Santa Cruz, Physics Department, Santa Cruz, California 95064 USA \\ ${ }^{4}$ Michigan Center for Theoretical Physics, Physics Department, University of Michigan, Ann Arbor, Michigan 48109, USA \\ ${ }^{5}$ Astronomy and Astrophysics Department, University of Chicago, Chicago, Illinois 60637 USA \\ ${ }^{6}$ Department of Physics, University of California, Berkeley, California 94720, USA \\ ${ }^{7}$ Theoretical Physics Group, LBNL, Berkeley, California 94720, USA \\ ${ }^{8}$ IPMU, University of Tokyo, 5-1-5 Kashiwa-no-ha, Kashiwa, Japan 277-8568
}

(Received 4 August 2009; revised manuscript received 20 November 2009; published 13 January 2010)

It has been suggested that the excesses of high-energy cosmic ray electrons and positrons seen by PAMELA and the Fermi Gamma Ray Space Telescope are evidence of dark matter annihilation or decay in the Galactic halo. To accommodate these signals however, the final states must be predominantly muons or taus. These leptonic final states will produce neutrinos, which are potentially detectable with the IceCube neutrino observatory. We find that with five years of data, IceCube (supplemented by DeepCore) can significantly constrain the relevant parameter space for both annihilating or decaying dark matter, and may be capable of discovering leptophilic dark matter in the halo of the Milky Way.

\section{INTRODUCTION}

A number of recent measurements of cosmic ray electrons and positrons have been interpreted as possible evidence for dark matter [1-6]. In particular, it has been suggested that the features of the $e^{+} e^{-}$spectrum and positron fraction reported by PAMELA [1], ATIC [2], PPB-BETS [3], the Fermi Gamma Ray Space Telescope (FGST) [4], HEAT [5], and AMS-01 [6] may originate from either DM annihilations [7-21] or decays [22-27] taking place in the Galactic Halo. As an aside, it should be noted that these signals may originate from astrophysical sources, rather than from new high energy physics. In particular, acceleration of electrons and positrons in the magnetic fields of nearby supernova remnants provide a compelling alternative candidate for the source of these anomalies $[28,29]$.

Although annihilating dark matter could potentially generate the observed anomalous cosmic ray features, attempts to do so face two main challenges. First, the spectrum of electrons and positrons predicted to be generated in the annihilations of most dark matter candidates is too soft to fit the observations of PAMELA and FGST $[10,11]$. If weakly interacting massive particles (WIMPs) annihilating throughout the halo of the Milky Way are to produce the spectral shape observed by these experiments they must annihilate mostly to charged leptons. While models have been proposed in which this is the case [12$21,30]$, many of the most often studied WIMP candidates (including MSSM neutralinos) are predicted to annihilate dominantly to quarks and/or gauge bosons [31]. ${ }^{1}$

\footnotetext{
${ }^{1}$ It should be noted that some exceptions have been put forth, for example, Ref. [32].
}

Furthermore, annihilations to nonleptonic final states tend to produce more cosmic ray antiprotons than are observed $[30,33]$.

Second, the dark matter annihilation rate that is required to generate the observed spectrum of cosmic ray electrons and positrons is considerably higher than is predicted for a typical thermal relic distributed smoothly throughout the Galactic Halo. To normalize the annihilation rate to the PAMELA and FGST signals, we must require either large inhomogeneities in the dark matter distribution which lead to a considerably enhanced annihilation rate (i.e. a "boost factor"), and/or dark matter particles which possess a considerably larger annihilation cross section than is necessary for a thermal relic. This latter possibility requires either a nonthermal production mechanism in the early universe, or an enhancement of the annihilation cross section at low velocities, possibly through the Sommerfeld effect [20,34] or a Breit-Wigner enhancement [35]. In light of these challenges, the observations from PAMELA and FGST are extremely surprising and pose an interesting challenge to the usual WIMP paradigm.

Neutrinos offer a unique opportunity to confirm or disprove a dark matter origin for these anomalous signals. The unusual features of the WMAP [36] as well as the FGST [37] haze, and the PAMELA and FGST excesses may well be explained by astrophysical sources [28,29]. Alternatively, some of the excess may be explained by large uncertainties in GALPROP [38]. FGST and PAMELA can both be well explained by supernovae and a known population of Pulsars $[28,29,39]$. The WMAP haze and the FGST haze must deal with multiple backgrounds. Luckily, a DM neutrino signal avoids many of these complications. There are no known astrophysical backgrounds which could fake a DM signal in the neutrino channel. 
Furthermore, the only background (from atmospheric neutrinos) is both well understood and not overwhelming. As a result, neutrino searches may prove a clean signature of DM annihilation or decay.

In this paper, we consider searches for associated production of neutrinos originating either from DM annihilation or decay at the IceCube neutrino observatory supplemented by the planned extension DeepCore. While neutrinos from WIMP annihilations in the halo of our galaxy have been studied previously [40], there are new implications for this detection channel in light of the high annihilation or decay rate and preference for leptonic modes required to explain the PAMELA and other anomalous cosmic ray signals. Furthermore, decays or annihilations directly into $e^{+} e^{-}$final states are disfavored by the observed spectra, requiring instead muon or tau final states. It has been shown that dark matter with a mass $\sim 10^{2-5} \mathrm{GeV}$ that annihilates/decays into $\mu^{-} \mu^{+}, \tau^{-} \tau^{+}$, or $\mu^{-} \mu^{+} \mu^{-} \mu^{+}$can reproduce the observed cosmic ray features [11]. This results in copious production of neutrinos as the heavy leptons decays. There is also the possibility that some dark matter goes directly to neutrino final states, rather than charged leptons, which would serve to increase the sensitivity of such a search.

The addition of DeepCore (expected to be completed in 2010) within the IceCube detector will also improve this search, as DeepCore allows IceCube to observe neutrinoinduced showers with energy in the range of tens of $\mathrm{GeV}$. Without DeepCore, using IceCube to study the inner Milky Way is difficult because of the large background of down-going atmospheric muons. DeepCore allows the IceCube detector itself to be used as a veto for muon backgrounds, making the identification of neutrinoinduced showers from the DM annihilations in the Galactic center possible [41]. Using this technique, it is possible to place bounds on the properties of either annihilating or decaying dark matter in the regions of parameter space required to explain PAMELA/FGST.

Only a few phenomenological parameters are relevant to the search for neutrino signals of DM in the Galactic center. Whether DM is decaying [22-27] or annihilating provides a clear division, and we consider both possibilities separately. In the latter case, the DM mass, cross section, and annihilation branching ratios are the only model inputs needed; though we choose to assume a canonical WIMP cross section obtained from thermal freeze out in the early universe and instead parametrize our lack of knowledge in terms of a boost factor $B$. Similarly, our search for decaying dark matter requires only an input of mass, lifetime $\tau$, and decay channels. Beyond these few properties, the nature of the dark matter particles is not important to our study, and the obtained limits are correspondingly robust.

The remainder of the paper is divided into three sections. In Sec, II, we show the discovery reach of IceCube/ DeepCore for annihilating dark matter, in terms of the boost factors to which we are sensitive to after five years of observation (for given mass and final states). In Sec. III, we do the same for decaying DM. In this case we place limits on the lifetime, rather than a boost factor. In both cases, we find that we can probe annihilation or decay parameters in the ranges of interest for the PAMELA/ FGST anomalies. We conclude in Sec. IV.

\section{ANNIHILATING DARK MATTER}

The flux of DM annihilation products from the direction of the Galactic center is given by

$$
\frac{d \Phi(\Delta \Omega, E)}{d E}=\frac{B}{8 \pi} \frac{\langle\sigma v\rangle}{m_{\chi}^{2}} \bar{J}(\Delta \Omega) \Delta \Omega \sum_{i} f_{i} \frac{d N_{\nu}^{i}}{d E_{\nu}},
$$

where $f_{i}$ is the branching ratio to a given species, $d N_{\nu}^{i} / d E_{\nu}$ is the differential neutrino spectrum per annihilating WIMP, $B$ is the boost factor, $m_{\chi}$ is the DM mass, and $\langle\sigma v\rangle$ the annihilation cross section. The DM distribution integrated over the line-of-sight over a solid angle, $\Delta \Omega$, is given by

$$
J=\int_{\text {l.o.s. }} \rho_{\chi}^{2}(s) d s ; \quad \bar{J}(\Delta \Omega)=\frac{1}{\Delta \Omega} \int_{\Delta \Omega} \operatorname{PSF} \star J d \Omega,
$$

where PSF is the point spread function of the instrument and the $\star$ operator represents convolution of the line-ofsight integral over the PSF. Because of the poor resolution of IceCube we have treated the Galactic Center as a point source. As a result we set the PSF to unity. Also, throughout our study, we will assume $\langle\sigma v\rangle=3 \times 10^{-26} \mathrm{~cm}^{3} / \mathrm{s}$ (the standard estimate for the DM annihilation cross section for a WIMP that is thermally produced in the early universe).

The spectrum of neutrinos resulting from a WIMP annihilation depends on the mass of the WIMP and on the dominant annihilation modes. WIMPs annihilating to muons produce muon and electron neutrinos in their decays. Annihilations to taus produce neutrinos through a variety of decay processes, including the leptonic modes $\tau \rightarrow \mu \nu \nu, e \nu \nu$, as well as from the hadronic decays $\tau \rightarrow$ $\pi \nu, K \nu, \pi \pi \nu$, and $\pi \pi \pi \nu$ [42,43]. Decays directly to $\nu \bar{\nu}$ of course produce a monoenergetic neutrino spectrum. For this work, the spectrum of neutrinos from tau decays as a function of $m_{\chi}$ was determined numerically using PYTHIA [44]. As the FGST and PAMELA anomalies are in the electron/positron channels, it is natural to consider DM annihilation directly to $e^{+} e^{-}$, which obviously produces no neutrinos. However, numerous studies (e.g. [11,30,33]) have found that electron/positron final states are disfavored compared to decays to heavy charged leptons, as the former produces too many hard electrons in the cosmic ray spectrum.

The primary backgrounds consist of atmospheric muons and neutrinos. The IceCube detector itself can be used to 
TABLE I. The capability of IceCube/DeepCore to detect neutrino-induced showers in a scenario in which the PAMELA and FGST signals are both the result of DM annihilations. We show results for DM which annihilates either entirely to muons or to an equal number of muons and muon neutrinos. The limits on the boost factor and the discovery prospects given correspond to five years of observation. The boost factors required to normalize to the PAMELA and FGST signals are inferred from Ref. [11].

\begin{tabular}{lcccccc}
\hline \hline $\begin{array}{l}m_{\chi} \\
(\mathrm{GeV})\end{array}$ & $\begin{array}{c}\text { Bin Size } \\
(\mathrm{GeV})\end{array}$ & $\begin{array}{c}5 \sigma \text { Detection } \\
\left(\mu^{+} \mu^{-}\right)\end{array}$ & $\begin{array}{c}2 \sigma \text { Limit } \\
\left(\mu^{+} \mu^{-}\right)\end{array}$ & $\begin{array}{c}5 \sigma \text { Detection } \\
\left(\mu^{+} \mu^{-}+\nu_{\mu} \bar{\nu}_{\mu}\right)\end{array}$ & $\begin{array}{c}2 \sigma \text { Limit } \\
\left(\mu^{+} \mu^{-}+\nu_{\mu} \bar{\nu}_{\mu}\right)\end{array}$ & $\begin{array}{c}\text { Norm. Required by PAMELA+FGST } \\
\left(\mu^{+} \mu^{-}\right. \\
\text {case })\end{array}$ \\
\hline 2000 & $600-3000$ & $B \geq 570$ & $B \leq 230$ & $B \geq 350$ & $B \leq 140$ & $B \approx 1700$ \\
1000 & $1500-300$ & $B \geq 430$ & $B \leq 170$ & $B \geq 270$ & $B \leq 110$ & $B \approx 450-700$ \\
\hline \hline
\end{tabular}

TABLE II. The capability of IceCube/DeepCore to detect neutrino-induced showers in a scenario in which the PAMELA signal is the result of DM annihilations. We do not attempt to fit the FGST result. The PAMELA boost factors are obtained from Ref. [10].

\begin{tabular}{|c|c|c|c|c|c|c|c|c|}
\hline $\begin{array}{l}m_{\chi} \\
(\mathrm{GeV})\end{array}$ & $\begin{array}{l}\text { Bin Size } \\
(\mathrm{GeV})\end{array}$ & $\begin{array}{l}\sigma \text { Detection } \\
\left(\mu^{+} \mu^{-}\right)\end{array}$ & $\begin{array}{l}2 \sigma \text { Limit } 5 \\
\left(\mu^{+} \mu^{-}\right)\end{array}$ & $\begin{array}{c}\sigma \text { Detectio } \\
\left(\tau^{+} \tau^{-}\right)\end{array}$ & $\begin{array}{l}2 \sigma \text { Limit } \\
\left(\tau^{+} \tau^{-}\right)\end{array}$ & $\begin{array}{c}5 \sigma \text { Detection } \\
\left(\mu^{+} \mu^{-}+\nu_{\mu} \bar{\nu}_{\mu}\right.\end{array}$ & $\begin{array}{l}2 \sigma \text { Limit } \\
\left.\mu^{-}+\nu_{\mu} \bar{\nu}_{\mu}\right)\end{array}$ & Norm. Required by PAMELA \\
\hline 500 & $150-800$ & $B \geq 320$ & $B \leq 130$ & $B \geq 480$ & $B \leq 190$ & $B \geq 200$ & $B \leq 80$ & $\begin{aligned} B & \approx 120-800\left(\mu^{+} \mu^{-}\right) \\
B & \approx 200-500\left(\tau^{+} \tau^{-}\right)\end{aligned}$ \\
\hline 300 & $100-500$ & $B \geq 260$ & $B \leq 100$ & $B \geq 370$ & $B \leq 150$ & $B \geq 159$ & $B \leq 60$ & $\begin{array}{c}B \approx 40-180\left(\mu^{+} \mu^{-}\right) \\
B \approx 70-160\left(\tau^{+} \tau^{-}\right)\end{array}$ \\
\hline 150 & $50-250$ & $B \geq 190$ & $B \leq 70$ & $B \geq 270$ & $B \leq 110$ & $B \geq 110$ & $B \leq 40$ & $B \approx 10-50\left(\mu^{+} \mu^{-}\right) \mathrm{NA}\left(\tau^{+} \tau^{-}\right)$ \\
\hline
\end{tabular}

veto muons inside of the volume of DeepCore, leaving only neutrino-induced showers to compete with. For the spectrum of atmospheric neutrinos, we use the results of Refs. [45-47], which are in good agreement with the measurements of AMANDA [48]. ${ }^{2}$

The effective area of the detector for neutrinos can be defined as

$$
A(E) \approx \rho_{\mathrm{ice}} N_{A} \sigma_{\nu N}(E) V(E)
$$

where $\rho_{\text {ice }}=0.9 \mathrm{~g} / \mathrm{cm}^{3}, N_{A}=6.022 \times 10^{23} \mathrm{~g}^{-1}$ (to convert grams to nucleons), $\sigma_{\nu N}(E)$ is the neutrino-nucleon cross section [49], and $V(E)$ is the effective volume of the detector for a neutrino-induced shower of energy $E$. However, for the energy range of interest we may approximate this volume as energy independent, with a value of $V(E) \approx 0.04 \mathrm{~km}^{3}[41]$.

The directional capability of IceCube for a neutrinoinduced shower above $1 \mathrm{TeV}$ is expected to be on the order of $50^{\circ}$ [41]. We conservatively consider the signal and background over a solid angle corresponding to a full half of the sky $(2 \pi \mathrm{sr})$, acknowledging that our results would be strengthened if better angular resolution could be obtained. Using a NFW profile, we integrate the DM distribution in the direction of the Galactic center over this

\footnotetext{
${ }^{2}$ As the $\nu_{\mu}$ background is considerably larger than that from $\nu_{e}$ 's, any discrimination between electromagnetic and hadronic showers could be used to further reduce the backgrounds and improve the statistical reach of IceCube/Deepcore to the signal described in this paper.
}

solid angle. We take the energy resolution of the detector to be $\log \left(E_{\text {max }} / E_{\text {min }}\right) \sim 0.3$ [41].

As a first case, we consider a dark matter particle $\chi$ which can potentially provide both the rising positron fraction observed by PAMELA [1] and electron spectrum observed by FGST [4]. This requires $m_{\chi} \gtrsim 1 \mathrm{TeV}$, and annihilations preferentially to muons (as opposed to electrons or taus) [11]. In Table I we show the boost factors to the annihilation rate that would be required discovery or exclusion by IceCube/DeepCore, and compare to the boost factor that would be required to produce the PAMELA and FGST signals [11]. We find that DM candidates capable of generating the FGST and PAMELA signals will also be well within the reach of IceCube/Deepcore.

Next, we turn our attention to a DM particle capable of generating the positron excess observed by PAMELA, without requiring that it also produces the spectrum reported by FGST. This allows lower mass candidates, as well as annihilations to taus [10]. The results are shown in Table II. In this case, although the full range of annihilation channels and masses capable of providing the PAMELA signal cannot be tested by IceCube/DeepCore, a significant fraction of the models can be. In particular, a $500 \mathrm{GeV} \mathrm{DM}$ particle which annihilates largely to muons or taus will be near or within the $2 \sigma$ reach of IceCube/DeepCore for the entire range of boost factors capable of producing the PAMELA signal (this range corresponds to uncertainties in the cosmic ray propagation model). If the DM also has an annihilation channel directly to neutrinos, the reach is further extended. Lighter particles are more difficult for IceCube/DeepCore to constrain or detect. We summarize the constraints for the muon channels in Fig. 1. 


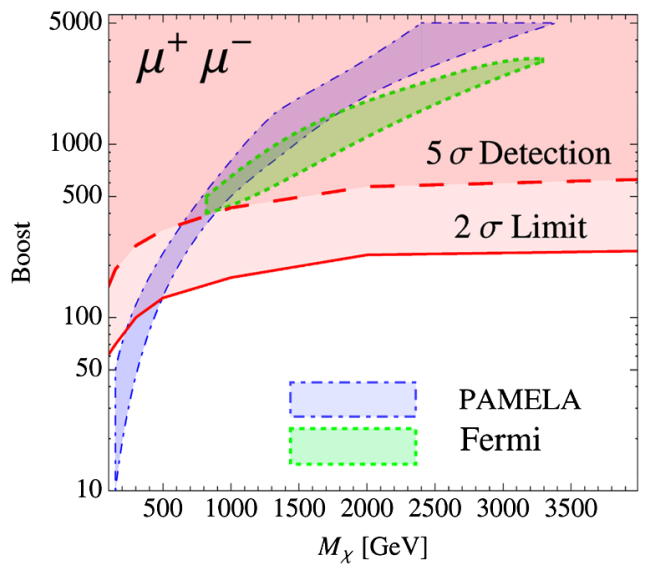

FIG. 1 (color online). Reach of ICECUBE/DeepCore to neutrinos from DM annihilation to $\mu^{+} \mu^{-}$. Shown are the $5 \sigma$ (dashed line) detection and the $2 \sigma$ limit (solid line) on the boost factor as a function of WIMP mass after 5 years of operation. Also shown are the $2 \sigma$ contours in the boost factor $B$ as function of DM mass for Fermi (dotted line) and PAMELA (dot-dashed line) inferred from [11] for $\left(\chi \chi \rightarrow \mu^{+} \mu^{-}\right)$.

\section{DECAYING DARK MATTER}

As outlined in the introduction, the lack of a signal in the antiproton channel in association with the electron/positron excesses observed by PAMELA and FGST, in combination with the unusually large annihilation cross section required pose difficulties for a standard WIMP dark matter candidate. While the annihilations to heavy charged leptons with large boost factors explored in the previous section are a possibility, if we are forced to move beyond the straightforward story which motivated the WIMP paradigm, decaying dark matter is an intriguing possibility [2226]. Decaying dark matter avoids the need for a boost factor, although the long lifetime of the DM (on the order of $10^{26} \mathrm{~s}$ ) replaces it is as an arbitrary parameter that needs explanation. However, the possibility that DM is only meta-stable has opened a rich new area of possible phenomenology and model-building, and so the possibility of constraining this sector is an interesting and relevant goal.

In the case of decaying DM, the neutrino flux calculation proceeds very similarly as in Sec. II, with one exception that deserves comment. In the case of annihilation, the rate goes as local density squared, as seen in the definition of $\bar{J}$ in Eq. (2). However, when decaying, the equivalent quantity, which we call $\overline{\mathcal{J}}$, depends on only power of the density:

$$
\begin{aligned}
\mathcal{J} & =\int_{\text {l.o.s. }} \rho_{\chi}(s) d s ; \\
\overline{\mathcal{J}}(\Delta \Omega) & =\frac{1}{\Delta \Omega} \int_{\Delta \Omega} \operatorname{PSF} \star \mathcal{J} d \Omega .
\end{aligned}
$$

As before, PSF is the point spread function of the instrument and $\rho_{\chi}(s)$ is the dark matter mass density distribu- tion. As in Sec. II we take the point spread function to be unity.

With this caveat, the flux of neutrinos from dark matter decay in the inner Milky Way is given by

$$
\frac{d \Phi(\Delta \Omega, E)}{d E}=\frac{1}{4 \pi} \frac{\Gamma}{m_{\chi}} \overline{\mathcal{J}}(\Delta \Omega) \Delta \Omega \sum_{i} \frac{d N_{i}}{d E}
$$

Here, $\Gamma=\tau^{-1}$ is the decay width of the dark matter, $m_{\chi}$ is the dark matter mass, and $d N_{i} / d E$ is the differential flux of neutrinos of flavor $i$ resulting from the decay, and as stated the dark matter distribution integrated over the line-ofsight over a solid angle $\Delta \Omega$ is $\mathcal{J}$.

Similar to the case of annihilation, the spectrum of neutrinos depends on the available channels available to the leptonic final states of the dark matter decay. We once again used PYTHIA to numerically simulate the neutrino spectrum for muon and tau decay in the $\mu^{+} \mu^{-}$, $\mu^{+} \mu^{-} \mu^{+} \mu^{-}$, and $\tau^{+} \tau^{-}$cases. It should be noted that for the four muon final state, it is assumed that the two $\mu^{+} \mu^{-}$pairs originate from the decays of back-to-back parent particles. As a result, the rest energy $m_{\chi}$ is split evenly between the two pairs, rather than distributed among the four particles as per naive phase-space.

The background rate of atmospherical neutrinos is calculated as in Sec. II. As before, we integrate over $2 \pi$ sr of solid angle towards the Galactic center, assuming a NFW profile. Binning is done assuming a detector resolution of $\log \left(E_{\max } / E_{\min }\right) \sim 0.3$. We assume that the excess of the $\operatorname{cosmic} e^{+}+e^{-}$spectrum measured by FGST beyond the simple power law is the dark matter decay signal (For alternative interpretations, see, e.g., [39]).

For dark matter masses between $100 \mathrm{GeV}$ and $30 \mathrm{TeV}$, we calculate the lifetime for which IceCube/DeepCore would provide either a $2 \sigma$ exclusion limit or a $5 \sigma$ discovery after five years of observation. The limits for the three decay channels under consideration are shown in Table III. In Fig. 2, we overlay our results on top of the regions of $m_{\chi}$ vs $\tau$ parameter space preferred by PAMELA/FGST. Also shown are the regions of parameter space excluded by HESS and VERITAS [50-54] as well as the limits from Super-Kamiokande [55,56], FGST observations of gamma rays from inverse Compton scattering below $10 \mathrm{GeV}$, and radio observations of the Galactic center. In the figure, our results (blue and red $2 \sigma$ and $5 \sigma$ lines from IceCube/ DeepCore) have been superimposed upon a plot taken from Ref. [27] with permission of the authors. In this plot, the dwarf spheroidal (dS- $\gamma$ ) limit was originally determined in Ref. [57].

A noticeable dip in sensitivity is found for the case of a $\sim 100 \mathrm{GeV}$ dark matter particle decaying to four muons. In this case, the maximum energy carried away by each muon is not much larger than the energy threshold of IceCube/ DeepCore, approximately $20 \mathrm{GeV}$. As a result, most of the neutrinos from such decays are unobservable. 
TABLE III. Limits on lifetime, $\tau$, of dark matter decaying into $\mu^{+} \mu^{-}$(top), $\tau^{+} \tau^{-}$(middle), or $\mu^{+} \mu^{-} \mu^{+} \mu^{-}$(bottom) after 5 years of data collection at IceCube. Limits are placed for a discovery at $5 \sigma$ as well as a bound for $2 \sigma$ exclusion.

\begin{tabular}{|c|c|c|c|}
\hline$m_{\chi}(\mathrm{GeV})$ & Bin Size $(\mathrm{GeV})$ & $5 \sigma$ Detection $\left(\chi \rightarrow \mu^{+} \mu^{-}\right)$ & $2 \sigma$ Exclusion $\left(\chi \rightarrow \mu^{+} \mu^{-}\right)$ \\
\hline 100 & $20-250$ & $\tau<0.11 \times 10^{26} \mathrm{~s}$ & $\tau<0.28 \times 10^{26} \mathrm{~s}$ \\
\hline 150 & $20-250$ & $\tau<0.15 \times 10^{26} \mathrm{~s}$ & $\tau<0.38 \times 10^{26} \mathrm{~s}$ \\
\hline 300 & $20-250$ & $\tau<0.18 \times 10^{26} \mathrm{~s}$ & $\tau<0.45 \times 10^{26} \mathrm{~s}$ \\
\hline 500 & $100-500$ & $\tau<0.26 \times 10^{26} \mathrm{~s}$ & $\tau<0.65 \times 10^{26} \mathrm{~s}$ \\
\hline 1000 & $150-800$ & $\tau<0.40 \times 10^{26} \mathrm{~s}$ & $\tau<1.0 \times 10^{26} \mathrm{~s}$ \\
\hline 2000 & $300-1500$ & $\tau<0.55 \times 10^{26} \mathrm{~s}$ & $\tau<1.4 \times 10^{26} \mathrm{~s}$ \\
\hline 10000 & $1500-8000$ & $\tau<1.0 \times 10^{26} \mathrm{~s}$ & $\tau<2.7 \times 10^{26} \mathrm{~s}$ \\
\hline 30000 & $5000-25000$ & $\tau<1.4 \times 10^{26} \mathrm{~s}$ & $\tau<3.9 \times 10^{26} \mathrm{~s}$ \\
\hline$m_{\chi}(\mathrm{GeV})$ & Bin Size $(\mathrm{GeV})$ & $5 \sigma$ Detection $\left(\chi \rightarrow \tau^{+} \tau^{-}\right)$ & $2 \sigma$ Exclusion $\left(\chi \rightarrow \tau^{+} \tau^{-}\right)$ \\
\hline 100 & $20-250$ & $\tau<0.099 \times 10^{26} \mathrm{~s}$ & $\tau<0.25 \times 10^{26} \mathrm{~s}$ \\
\hline 150 & $20-250$ & $\tau<0.13 \times 10^{26} \mathrm{~s}$ & $\tau<0.34 \times 10^{26} \mathrm{~s}$ \\
\hline 300 & $20-250$ & $\tau<0.17 \times 10^{26} \mathrm{~s}$ & $\tau<0.43 \times 10^{26} \mathrm{~s}$ \\
\hline 500 & $100-500$ & $\tau<0.23 \times 10^{26} \mathrm{~s}$ & $\tau<0.57 \times 10^{26} \mathrm{~s}$ \\
\hline 1000 & $150-800$ & $\tau<0.34 \times 10^{26} \mathrm{~s}$ & $\tau<0.87 \times 10^{26} \mathrm{~s}$ \\
\hline 2000 & $300-1500$ & $\tau<0.47 \times 10^{26} \mathrm{~s}$ & $\tau<1.2 \times 10^{26} \mathrm{~s}$ \\
\hline 10000 & $1500-8000$ & $\tau<1.0 \times 10^{26} \mathrm{~s}$ & $\tau<2.7 \times 10^{26} \mathrm{~s}$ \\
\hline 30000 & $5000-25000$ & $\tau<1.2 \times 10^{26} \mathrm{~s}$ & $\tau<3.3 \times 10^{26} \mathrm{~s}$ \\
\hline$m_{\chi}(\mathrm{GeV})$ & Bin Size $(\mathrm{GeV})$ & $5 \sigma$ Detection $\left(\chi \rightarrow \mu^{+} \mu^{-} \mu^{+} \mu^{-}\right)$ & $2 \sigma$ Exclusion $\left(\chi \rightarrow \mu^{+} \mu^{-} \mu^{+} \mu^{-}\right)$ \\
\hline 100 & $20-250$ & $\tau<0.0081 \times 10^{26} \mathrm{~s}$ & $\tau<0.021 \times 10^{26} \mathrm{~s}$ \\
\hline 150 & $20-250$ & $\tau<0.068 \times 10^{26} \mathrm{~s}$ & $\tau<0.17 \times 10^{26} \mathrm{~s}$ \\
\hline 300 & $20-250$ & $\tau<0.11 \times 10^{26} \mathrm{~s}$ & $\tau<0.27 \times 10^{26} \mathrm{~s}$ \\
\hline 500 & $20-250$ & $\tau<0.18 \times 10^{26} \mathrm{~s}$ & $\tau<0.44 \times 10^{26} \mathrm{~s}$ \\
\hline 1000 & $100-500$ & $\tau<0.25 \times 10^{26} \mathrm{~s}$ & $\tau<0.65 \times 10^{26} \mathrm{~s}$ \\
\hline 2000 & $150-800$ & $\tau<0.40 \times 10^{26} \mathrm{~s}$ & $\tau<1.0 \times 10^{26} \mathrm{~s}$ \\
\hline 10000 & $600-3000$ & $\tau<0.82 \times 10^{26} \mathrm{~s}$ & $\tau<2.1 \times 10^{26} \mathrm{~s}$ \\
\hline 30000 & $1500-8000$ & $\tau<1.2 \times 10^{26} \mathrm{~s}$ & $\tau<3.2 \times 10^{26} \mathrm{~s}$ \\
\hline
\end{tabular}
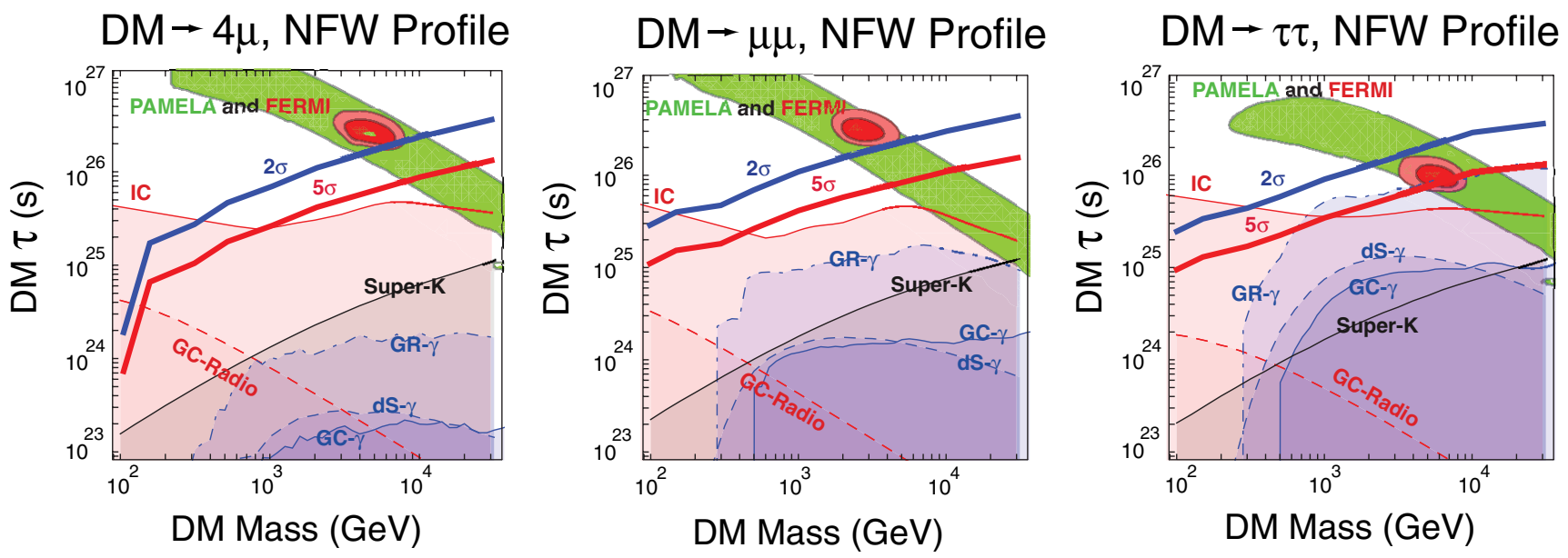

FIG. 2 (color online). Exclusion limits in the dark matter lifetime versus mass plane, for decays to $\mu^{+} \mu^{-}, \tau^{+} \tau^{-}$, and $\mu^{+} \mu^{-} \mu^{+} \mu^{-}$. The green region is preferred by the observations of PAMELA, light red is preferred by FGST, and the dark red region is preferred by the combination of these measurements. The blue line labeled " $2 \sigma$ " is the projected $2 \sigma$ limit for 5 -years of running at IceCube/ DeepCore, while the red " $5 \sigma$ " line is the $5 \sigma$ discovery reach. For details of the other bounds displayed, see text. Our results (blue $2 \sigma$ and red $5 \sigma$ lines from IceCube/DeepCore) have been superimposed upon a plot taken from Ref. [27] with permission of the authors. 


\section{CONCLUSIONS}

In the annihilation channel, we find that, in any scenario in which dark matter annihilations produce both the PAMELA and FGST signals, IceCube/DeepCore should be capable of detecting corresponding neutrinos with greater than $5 \sigma$ significance. In a scenario in which only the PAMELA excess results from dark matter annihilations, IceCube/DeepCore will be capable of placing stringent constraints, but will likely not be able to exclude the entire range of possible dark matter masses and annihilation modes.

From the results of Fig. 2, we see that IceCube, including the DeepCore extension, can probe the most interesting regions of the $m_{\chi}-\tau$ parameter space with five years of data after the planned completion in 2010. Because of its vastly larger volume, IceCube can probe dark matter decay lifetimes that are orders of magnitude greater than those excluded by Super-Kamiokande. IceCube/DeepCore will be competitive with or more constraining than inverse Compton and $\gamma$-ray measurements, and will serve as a complementary test of the dark matter interpretation of PAMELA and FGST results.

Regardless, bounds from IceCube are potentially more robust than alternative methods. Many experiments (HESS, Veritas, WMAP, EGRET, etc.) have been used to constrain the large DM parameter space (mass and boost or lifetime), which can feasibly explain the PAMELA and FGST anomalies. These constraints arise by requiring that a DM signal not overwhelm known backgrounds (such as the $\gamma$-ray background from EGRET or X-rays [58]), disturb the CMB [59], or over produce synchrotron radiation or $\gamma$-rays from the galactic center as discussed in Ref. [27] and shown in Fig. 2.

However, these experiments must make assumptions about unknown parameters, such as the DM profile, the detailed energy deposition from DM annihilation at the time of recombination, or the magnetic field structure in the Milky Way. Bounds from neutrinos are less sensitive to these complexities. They depend only weakly upon the DM profile and are independent of the other constraints. The neutrino background is well modeled [45-47] and is already well constrained by AMANDA [48]. IceCube will only improve these constraints. Hence, if no DM signal is found at IceCube, leptonic DM will be strongly constrained.

It should be noted that our results could potentially be improved upon if the angular resolution of IceCube/ DeepCore turns out to be considerably better than we have assumed here. Whereas the backgrounds are distributed broadly over the entire solid angle considered, the signal is concentrated in the region around the Galactic center, thus enabling much greater statistical power if the angular window were to be reduced. Furthermore, as the $\nu_{\mu}$ background is considerably larger than that from $\nu_{e}$, any discrimination between electromagnetic and hadronic showers could be used to reduce the backgrounds and improve the statistical reach of IceCube/Deepcore to the signal discussed here. Although we have assumed that such a discrimination is not possible, we remain hopeful that this will improve in the future.

Several effects may modify our results by factors $\mathcal{O}(1)$. [11] examined constraints on leptophilic annihilating DM due to HESS [52] $\gamma$ observations of the Galactic Ridge. They argue that NFW is not a good fit to this data; instead an isothermal sphere matches the data better (though not found in numerical simulations of galaxy formation). If we were to redo our analysis with an isothermal sphere, our results on the boost factor would be weaker by roughly a factor of 2. We note that the work of [11] that we used in our figure also assumed an isothermal sphere. However, in this case the difference in profiles is irrelevant because the PAMELA and FERMI data are sensitive only to the local dark matter density.

Specific to the annihilation mode, we have not included the additional boost due to substructure in the Milky Way, which is undoubtedly present and increases the signal $\mathcal{O}(1)$ [60]. Also, [52] would not be sensitive to this extra boost, since very little substructure exists at the center of the galaxy [60]. It is also interesting to note that, if the boost factor is due to a Sommerfeld effect, which is velocity dependent, the boost would be larger inside substructure (where the velocities are lower) [61].

\section{ACKNOWLEDGMENTS}

We would like to thank A. Aguirre, P. Gondolo, K. Hoffman, S. Profumo, F. Halzen, I. Mocioiu, and especially Spencer Klein for useful discussions. K. F. is supported by the U.S. Department of Energy and MCTP via the University of Michigan and the National Science Foundation under Grant No. PHY-0455649; D. S. is supported by NSF Grant No. AST-0507117 and GAANN (D.S.); D. H. is supported by the U.S. Department of Energy, including Grant No. DE-FG02-95ER40896, and by NASA Grant No. NAG5-10842; M. R. B. is supported by the Department of Energy, under Grant No. DE-FG0392-ER40701. H. M. is supported in part by World Premier International Research Center Initiative (WPI Initiative), MEXT, Japan, in part by the U.S. DOE under Contract No. DE-AC03-76SF00098, and in part by the NSF under Grant No. PHY-04-57315. The authors would also like to thank the Aspen Center for Physics for providing a stimulating atmosphere for research and collaboration. D.S. would also like to thank the MCTP. 
[1] O. Adriani et al. (PAMELA), Nature (London) 458, 607 (2009).

[2] J. Chang et al., Nature (London) 456, 362 (2008).

[3] S. Torii et al. (PPB-BETS), arXiv:0809.0760.

[4] A. A. Abdo et al. (Fermi LAT), Phys. Rev. Lett. 102, 181101 (2009).

[5] S. W. Barwick et al. (HEAT), Astrophys. J. 482, L191 (1997).

[6] M. Aguilar et al. (AMS-01), Phys. Lett. B 646, 145 (2007).

[7] E. A. Baltz, J. Edsjo, K. Freese, and P. Gondolo, Phys. Rev. D 65, 063511 (2002).

[8] V. Barger, W. Y. Keung, D. Marfatia, and G. Shaughnessy, Phys. Lett. B 672, 141 (2009).

[9] M. Cirelli and A. Strumia, arXiv:0808.3867.

[10] I. Cholis, L. Goodenough, D. Hooper, M. Simet, and N. Weiner, Phys. Rev. D 80, 123511 (2009).

[11] L. Bergstrom, J. Edsjo, and G. Zaharijas, Phys. Rev. Lett. 103, 031103 (2009).

[12] C.-R. Chen and F. Takahashi, J. Cosmol. Astropart. Phys. 02 (2009) 004.

[13] A. E. Nelson and C. Spitzer, arXiv:0810.5167.

[14] I. Cholis, D. P. Finkbeiner, L. Goodenough, and N. Weiner, J. Cosmol. Astropart. Phys. 12 (2009) 007.

[15] R. Harnik and G. D. Kribs, Phys. Rev. D 79, 095007 (2009).

[16] P. J. Fox and E. Poppitz, Phys. Rev. D 79, 083528 (2009).

[17] I. Cholis, G. Dobler, D. P. Finkbeiner, L. Goodenough, and N. Weiner, Phys. Rev. D 80, 123518 (2009).

[18] K. M. Zurek, Phys. Rev. D 79, 115002 (2009).

[19] D. Hooper and T. M. P. Tait, Phys. Rev. D 80, 055028 (2009).

[20] N. Arkani-Hamed, D. P. Finkbeiner, T. R. Slatyer, and N. Weiner, Phys. Rev. D 79, 015014 (2009).

[21] D. Hooper and K. M. Zurek, Phys. Rev. D 79, 103529 (2009).

[22] E. Nardi, F. Sannino, and A. Strumia, J. Cosmol. Astropart. Phys. 01 (2009) 043.

[23] A. Arvanitaki et al., Phys. Rev. D 79, 105022 (2009).

[24] A. Arvanitaki et al., Phys. Rev. D 80, 055011 (2009).

[25] K. Ishiwata, S. Matsumoto, and T. Moroi, J. High Energy Phys. 05 (2009) 110.

[26] A. Ibarra, D. Tran, and C. Weniger, arXiv:0906.1571.

[27] P. Meade, M. Papucci, A. Strumia, and T. Volansky, arXiv:0905.0480.

[28] D. Hooper, P. Blasi, and P. D. Serpico, J. Cosmol. Astropart. Phys. 01 (2009) 025.

[29] S. Profumo, arXiv:0812.4457.

[30] M. Cirelli, M. Kadastik, M. Raidal, and A. Strumia, Nucl. Phys. B813, 1 (2009).

[31] J. R. Primack, D. Seckel, and B. Sadoulet, Annu. Rev. Nucl. Part. Sci. 38, 751 (1988).

[32] G. Kane, R. Lu, and S. Watson, Phys. Lett. B 681, 151 (2009).
[33] F. Donato, D. Maurin, P. Brun, T. Delahaye, and P. Salati, Phys. Rev. Lett. 102, 071301 (2009).

[34] A. Sommerfeld (1931).

[35] M. Ibe, H. Murayama, and T. T. Yanagida, Phys. Rev. D 79, 095009 (2009).

[36] D. P. Finkbeiner, Astrophys. J. 614, 186 (2004).

[37] G. Dobler, D. P. Finkbeiner, I. Cholis, T. R. Slatyer, and N. Weiner, arXiv:0910.4583.

[38] T. Delahaye et al., Astron. Astrophys. 501, 821 (2009).

[39] D. Grasso et al. (FERMI-LAT), Astropart. Phys. 32, 140 (2009).

[40] H. Yuksel, S. Horiuchi, J. F. Beacom, and S. Ando, Phys. Rev. D 76, 123506 (2007).

[41] E. Resconi and f. t. I. Collaboration, Nucl. Instrum. Methods Phys. Res., Sect. A 602, 7 (2009).

[42] G. Jungman and M. Kamionkowski, Phys. Rev. D 51, 328 (1995).

[43] D. Grellscheid and P. Richardson, arXiv:0710.1951.

[44] T. Sjostrand, S. Mrenna, and P. Skands, J. High Energy Phys. 05 (2006) 026.

[45] J. G. Learned and K. Mannheim, Annu. Rev. Nucl. Part. Sci. 50, 679 (2000).

[46] G. D. Barr, T. K. Gaisser, P. Lipari, S. Robbins, and T. Stanev, Phys. Rev. D 70, 023006 (2004).

[47] M. Honda, T. Kajita, K. Kasahara, S. Midorikawa, and T. Sanuki, Phys. Rev. D 75, 043006 (2007).

[48] R. Abbasi et al. (IceCube), Phys. Rev. D 79, 102005 (2009).

[49] R. Gandhi, C. Quigg, M. H. Reno, and I. Sarcevic, Phys. Rev. D 58, 093009 (1998).

[50] G. Bertone, M. Cirelli, A. Strumia, and M. Taoso, J. Cosmol. Astropart. Phys. 03 (2009) 009.

[51] M. Vivier (HESS Collaboration), 44th Rencontres de Moriond, 2009.

[52] F. Aharonian et al. (HESS), Nature (London) 439, 695 (2006).

[53] F. Aharonian (HESS), Astropart. Phys. 29, 55 (2008).

[54] C. M. Hui and f. t. V. Collaboration, in Proceedings of the 4th International Meeting on High Energy Gamma-Ray Astronomy, edited by F. A. Aharonian, W. Hofmann, and F. Rieger, AIP Conf. Proc. No. 1085 (2009), p. 407.

[55] S. Desai et al. (Super-Kamiokande), Phys. Rev. D 70, 083523 (2004).

[56] S. Desai et al. (Super-Kamiokande), Astropart. Phys. 29, 42 (2008).

[57] R. Essig, N. Sehgal, and L. E. Strigari, Phys. Rev. D 80, 023506 (2009).

[58] S. Profumo and T. E. Jeltema, J. Cosmol. Astropart. Phys. 07 (2009) 020.

[59] T. R. Slatyer, N. Padmanabhan, and D. P. Finkbeiner, Phys. Rev. D 80, 043526 (2009).

[60] V. Springel et al., Nature (London) 456, 73 (2008).

[61] B. Robertson and A. Zentner, Phys. Rev. D 79, 083525 (2009). 